to unpleasant stimuli than the control participants. In fact, the BPD participants had significantly lower skin conductance responses and showed an absence of the fear-potentiated startle response, suggesting a general state of underarousal. On the emotion regulation task, both groups showed similar startle responses when instructed to maintain or suppress their emotions. These results do not support current theories of emotion dysregulation in BPD, suggesting instead that those with BPD are hyporesponsive to affective stimuli.

02-02

\section{Pituitary volume in adolescents with first-presentation borderline personality disorder}

\section{B Garner', AM Chanen', L Phillips², D Velakoulis³ SJ Wood ${ }^{3,4}$, HJ Jackson 1,2, C Pantelis ${ }^{3}$, PD McGorry}

'ORYGEN Research Centre, Department of Psychiatry, The University of Melbourne; ${ }^{2} S c h o o l$ of Behavioural Sciences, The University of Melbourne; ${ }^{3}$ Melbourne Neuropsychiatry Centre, Department of Psychiatry, The University of Melbourne, and Melbourne Health; and ${ }^{\mathrm{B}}$ Brain Research Institute, Austin and Repatriation Medical Centre, Melbourne, Australia

Background: Borderline personality disorder (BPD) might be associated with hypothalamic-pituitaryadrenal (HPA) axis dysregulation. Previous studies in adult BPD samples have been equivocal possibly because of confounding factors, such as chronicity of BPD and associated comorbidity. Studying firstpresentation adolescent BPD samples minimizes these confounds.

Methods: Twenty BPD (15 women, mean age 17.3 years) and 20 healthy control participants (15 women, mean age 19.0 years) underwent magnetic resonance imaging scanning. Pituitary gland volumes (PGVs) were estimated and compared between the groups and also within the BPD group, based upon exposure to childhood trauma.

Results: PGV did not differ between patients with BPD and controls $(P=0.6$; effect size $=0.19)$. Patients with BPD exposed to childhood trauma $(n=9)$ had smaller $(-18 \%)$ pituitaries compared with patients with BPD with no exposure to childhood trauma $(n=10$; $P=0.1$; Effect size $=0.74$ ).

Conclusions: These findings suggest that exposure to trauma, rather than BPD per se, might be associated with altered PGV, possibly reflecting HPA dysfunction.

\section{An MRI study of pituitary volume and suicidal behaviour in adolescents with first-presentation borderline personality disorder}

\author{
M Jovev', B Garner', L Phillips², D Velakoulis ${ }^{3}$, \\ SJ Wood ${ }^{3,4}$, HJ Jackson ${ }^{1,2}$, C Pantelis ${ }^{3}$, \\ PD McGorry', AM Chanen
}

\begin{abstract}
'ORYGEN Research Centre, Department of Psychiatry, The University of Melbourne; ${ }^{2}$ School of Behavioural Science, The University of Melbourne; ${ }^{3}$ Melbourne Neuropsychiatry Centre, Department of Psychiatry, The University of Melbourne \& Melbourne Health; and ${ }^{4}$ Brain Research Institute, Melbourne, Australia
\end{abstract}

There has been a lack of research examining the association between hypothalamic-pituitary-adrenal (HPA) axis function and suicidal behaviour in patients diagnosed with borderline personality disorder (BPD), despite the established relationship between BPD and suicide. This study aimed to investigate the relationship between pituitary gland volume (PGV) and number of suicidal behaviours in adolescent patients with first-presentation BPD. The main findings indicate that age, internalizing problems and number of suicidal behaviours are significant predictors of PGV in firstpresentation BPD. The larger PGV in those with a higher number of suicidal behaviours might reflect greater activation of the HPA axis by the actual act of selfharm or increased activation of the stress response by factors that also lead to suicidal behaviour, such as life events. The study points to the need to explore other suicide parameters (eg intent, medical threat) together with direct neuroendocrine measures in larger samples of patients with BPD.

\section{2-04}

\section{Interpersonal functioning in borderline personality disorder: the role of attachment, self-schema and social cognition}

\section{T Jennings', C Hulbert', H Jackson ${ }^{2}$, Andrew Chanen ${ }^{3}$}

'Austin Repatriation Hospital; ${ }^{2}$ School of Behavioural Science, The University of Melbourne; ORYGEN Research Centre, Parkville, Victoria, Australia

Background: The study investigated the clinical picture of young people with borderline personality disorder (BPD) traits by comparing them with a group of young people with major depressive disorder (MDD) on a set of variables that target core features of BPD 
and their etiology. In particular, the study used the interpersonal negotiation strategies (INS) model of social problem solving, alongside a measure of self-schema, to investigate potential maladaptive social-cognitive processes in BPD. Empirical findings regarding schema domains specific to BPD are limited and research into cognitive processes has focused on the single concept of dichotomous thinking.

Methods: Participants were 30 youths (15-24 years) with three or more BPD traits and 30 youths (15-24 years) with MDD. Participants received self-report measures of attachment, self-schema and interpersonal functioning. The INS interview assessed social perspective coordination skills in response to six hypothetical vignettes depicting situations of interpersonal conflict. To investigate the parameters of social information-processing biases, in $\mathrm{BPD}$, vignettes varied in terms of whether the conflict resembled BPD-specific schema content or neutral-schema content.

Results: The BPD group scored significantly higher on schemas of mistrust/abuse, insufficient self-control and entitlement and were characterized by high attachment anxiety and avoidance, but were differentiated from the MDD group by higher avoidance. The BPD group responded to interpersonal conflict with social perspective coordination skills of a significantly lower developmental level than the MDD group, regardless of vignette content. Young people with BPD traits were accordingly characterized by significantly worse interpersonal functioning.

Conclusion: The implications of these results for early cognitive intervention approaches to the treatment of $\mathrm{BPD}$ are discussed.

02-05

\section{The relationship of self-structure, attachment style and rejection sensitivity to borderline personality features}

\section{Hulbert, J Boldero, R Bell}

School of Behavioural Science, The University of Melbourne, Melbourne, Australia

Background: Views of self, others, relationships and social functioning form a significant part of the clinical picture of borderline personality disorder (BPD). Theoretical models have variously implicated temperament, most notably neuroticism, and aberrant attachment experience, along with related deficits in sense of self and social cognition in the etiology of this serious mental disorder. However, no research has systematically investigated the extent to which these views are associated with the characteristics of BPD. The present study examines the relationship of self-complexity and self-discrepancy, attachment style and rejection sensitivity to endorsement of BPD features.

Method: As part of course requirements, 101 first-year university students (70 women and 31 men) completed well-validated self-report measures of neuroticism, self-complexity and self-discrepancy, attachment style, rejection sensitivity and an 80 -item screening measure for BPD.

Results: With levels of neuroticism controlled for, significant positive relationships were found between attachment anxiety and avoidance, rejections sensitivity and endorsement of BPD features. In addition, the extent to which participants saw themselves as discrepant from their ideal and ought self-guides [actualideal (AI) and actual-ought (AO) self-discrepancies] in terms of both positive and negative self-roles and attributes were assessed, as was the extent to participants' self-concepts were complex with regard to the organization of positive and negative attributes. Reporting more BPD features was associated with large negative $\mathrm{AI}$ and $\mathrm{AO}$ discrepancies (reflecting greater possession of negative traits), as were the complexity of both negative roles and attributes. The data suggest that both the self-concept content and structure contribute to BPD.

Conclusion: The theoretical and clinical implications of the present findings and planned future research into the role of self and social cognition in BPD are discussed.

02-06

\section{Personality disorder in the elderly and the effect on outcome of comorbid axis I disorder}

\section{J Stevenson}

Westmead and Cumberland Hospitals and Sydney University, Sydney, Australia

Personality disorder in younger adult psychiatric inpatients is known to prolong the in-patient stay, impede recovery and increase health care costs. There is a significant association between the number of disordered personality traits and medical resource utilization. However, this has not been extensively studied in the elderly, where personality disorder is usually undiagnosed or ignored. It has previously been felt that personality disorder 'burns out' in old age, but there is no data supporting this. Our study is to determine the prevalence and types of personality disorder in elderly psychiatric in-patients and the impact if any it has 